\title{
Real-World Recurrence Rates and Economic Burden in Patients with Resected Early-Stage Melanoma
}

Sekwon Jang • Tayla Poretta • Tarun Bhagnani · Qing Harshaw •

Matthew Burke · Sumati Rao

Received: April 15, 2020 / Published online: June 16, 2020

(C) The Author(s) 2020, corrected publication 2020

\section{ABSTRACT}

Introduction: Real-world data on recurrence and economic burden in patients with resected early-stage melanoma are limited. The objective of this study was to assess real-world recurrence rates, risk factors for recurrence, and costs of recurrence in patients with resected stage IIB, IIC, or IIIA melanoma in the USA.

Methods: This retrospective analysis included patients with resected stage IIB, IIC, or IIIA melanoma (American Joint Committee on Cancer staging manual, seventh edition) in the Surveillance, Epidemiology, and End Results (SEER) program-Medicare database of the National Cancer Institute. Recurrence rates and healthcare costs (2018 USD) after recurrence were assessed.

Digital Features To view digital features for this article go to https://doi.org/10.6084/m9.figshare.12377624.

S. Jang ( $\square)$

Melanoma and Cutaneous Oncology Therapeutics, Inova Schar Cancer Institute, Fairfax, VA, USA

e-mail: Sekwon.Jang@inova.org

T. Poretta $\cdot$ M. Burke $\cdot$ S. Rao

Health Economics and Outcomes Research, Bristol Myers Squibb, Princeton, NJ, USA

T. Bhagnani · Q. Harshaw

Health Economics and Outcomes Research,

EPI-Q Inc., Oakbrook, IL, USA
Results: Two-year recurrence rates for stages IIB, IIC, and IIIA melanoma were 29, 44, and $46 \%$, respectively. In patients with stage IIB or IIC disease, the odds of recurrence were significantly higher in those aged $>75$ years [odds ratio (OR) 1.853, 95\% confidence interval (CI) 1.416, 2.425], with ulceration (OR 1.771; 95\% CI 1.293, 2.425), or with a higher Charlson Comorbidity Index (OR 1.244; 95\% CI 1.129, 1.372); however, the odds of recurrence were significantly lower in those with T3 staging (OR 0.522; 95\% CI 0.393, 0.695). In those with stage IIIA melanoma, superficial spreading was associated with significantly lower odds of recurrence (OR 0.178; 95\% CI 0.053, 0.601). Following recurrence, mean healthcare costs at 1 year were $\$ 31,870$ for patients with stage IIB or IIC melanoma and $\$ 29,224$ for those with stage IIIA melanoma.

Conclusion: The SEER data show that a substantial proportion of adults with early-stage melanoma experience a recurrence within 2 years following resection, resulting in a significant economic burden to the US healthcare system. Dermatologists can distinguish patients with resected early-stage melanoma who are at a high risk for recurrence and consider referrals to medical oncologists for approved adjuvant therapy or enrollment in clinical trials after surgical resection to reduce the recurrence of melanoma. 


\section{PLAIN LANGUAGE SUMMARY}

Melanoma is the sixth most common type of cancer in the USA. In the past several years, the US Food and Drug Administration has approved several novel therapies for patients with highrisk melanoma following surgery. However, these therapies are not approved for the treatment of patients with earlier-stage or intermediate-risk melanoma. In these patients, treatment choices include enrollment in clinical trials or observation. We have assessed recurrence rates, risk factors for recurrence, and costs of recurrence in patients with early-stage melanoma. This analysis included patients with resected early-stage melanoma in the US Surveillance, Epidemiology, and End Results (SEER) program-Medicare database. The results show that a substantial proportion of adults with early-stage melanoma experienced a recurrence within 2 years after surgical removal of their tumor, resulting in a significant economic burden to the healthcare system. Dermatologists can use information in the published literature to distinguish patients with resected early-stage melanoma who are at a high risk for recurrence and consider referrals to medical oncologists for approved therapy or enrollment in clinical trials after surgery to reduce recurrence and substantial economic consequences.

Keywords: Adjuvant; Early stage; Economic burden; Healthcare costs; Real-world; Recurrence rates; Resected melanoma; Skin cancer; Survival

\section{Key Summary Points}

\section{Why carry out this study?}

In the past several years, the US Food and Drug Administration and the European Medicines Agency have approved several novel therapies that demonstrated improvement in clinical outcomes and survival in patients with resected stage III or IV melanoma.
However, due to there currently being no approved novel immunotherapies or targeted therapies for patients with resected early-stage melanoma (stage IIB and IIC), enrollment in clinical trials or observation is the standard of care.

Given the limited data on the recurrence rates in patients with early-stage melanoma, the aim of this study was to assess real-world recurrence rates, risk factors for recurrence, and costs of recurrence in patients with resected stage IIB, IIC, or IIIA melanoma in the USA.

\section{What was learned from the study?}

Analysis of Surveillance, Epidemiology, and End Results (SEER)-Medicare linked data showed that a substantial proportion of US adults with early-stage melanoma experience a recurrence within 2 years following resection, associated with high healthcare resource use and costs, resulting in a significant economic burden to the US healthcare system.

Dermatologists can distinguish patients with resected early-stage melanoma who are at a high risk for recurrence and consider referrals to medical oncologists for approved adjuvant therapy or enrollment in clinical trials to reduce recurrence of melanoma and substantial economic consequences.

\section{INTRODUCTION}

Melanoma is the sixth most common type of cancer in the USA [1]. Surgical removal of the primary tumor followed by adjuvant therapy or observation is the recommended treatment for patients with stage III or IV melanoma who are at a high risk of recurrence [2]. In the past several years, the US Food and Drug Administration (FDA) has approved several novel therapies that were shown to improve clinical outcomes and survival in patients with resected stage III or IV melanoma [3-5]. 
Patients with early-stage melanoma, including those with thick or ulcerated primary lesions (stage IIB or IIC) and those with pathological or clinical evidence of regional nodal metastasis (stage III), also present a significant challenge, with 5-year survival rates ranging from 40 to $78 \%$ (staging based on the American Joint Committee on Cancer [AJCC] staging manual, seventh edition) [6]. Currently, only limited data, based on the AJCC staging manual, eighth edition (in which stages IIB and IIC are similar to those described in the AJCC, seventh edition) $[6,7]$, exist on recurrence rates in early-stage melanoma, and the reported rates vary. In one prospective study, patients with stage IIB and stage IIC disease exhibited 5-year recurrence-free survival (RFS) rates of $54.7 \%$ [95\% confidence interval (CI) 41.4\%, 72.3\%] and $26.5 \%$ (95\% CI $12.8 \%, 55.1 \%)$, respectively [8]. A retrospective study reported 5-year RFS rates for stage IIB and stage IIC patients to be $64.4 \%$ (95\% CI 55.5\%, 71.9\%) and 41.5\% (95\% CI $26.7 \%$, 55.7\%), respectively [9]. Another study, which used data from the German Central Malignant Melanoma Registry, reported 5 -year RFS rates of $65.5 \%$ for stage II and $56.9 \%$ for stage III melanoma [10]. In addition, 5-year melanoma-specific survival rates for patients with stages IIB and IIC have been reported to be 87 and $82 \%$, respectively [7].

Interferon alfa- $2 b$, the first immunotherapy approved by the FDA, was previously considered to be the standard of care for patients with resected stage IIB, stage IIC, or stage III melanoma [11], although it failed to demonstrate a consistent long-term survival benefit in this patient population [12-14]. An initial trial of high-dose interferon demonstrated significant RFS and overall survival (OS) benefits in the adjuvant setting [12]; however, a subsequent trial did not find significant OS benefits compared with observation (hazard ratio 1.0; $P=0.995$ ) [13]. While no longer recommended, interferon remains the only adjuvant therapy approved for patients with resected stage IIB or IIC melanoma. Enrollment in clinical trials or observation continue as the primary treatment choices for these patients [2]; however, patients with early-stage melanoma are frequently excluded from many clinical adjuvant therapy trials.

In the absence of data from randomized trials, real-world studies (analyses using data from cancer registries and administrative claims for patients not in clinical trials) can provide insights into the recurrence rates for patients with early-stage melanoma. To help address the paucity of data on recurrence in early-stage melanoma, we have assessed real-world recurrence rates, risk factors for recurrence, and costs following recurrence in US patients with resected stage IIB, IIC, or IIIA melanoma. In this retrospective study, a claims-based algorithm was used to define recurrence, making it necessary to collectively analyze different types of recurrence (i.e., local, regional, metastatic).

\section{METHODS}

\section{Data Source}

This retrospective analysis of the US Surveillance, Epidemiology, and End Results (SEER) program-Medicare linked database includes cancer registries on newly diagnosed cancer cases linked to administrative healthcare data (e.g., claims, eligibility for medical and pharmacy benefits, and demographic data). SEER is the largest population-based cancer registry, covering approximately $34 \%$ of the US population from 19 geographically disparate regions that are representative of the demographics of the national population [15]. Permission to use the SEER-Medicare data was obtained from the National Cancer Institute.

Individual patient-level data in the database comprise (1) primary and secondary diagnoses (in International Classification of Diseases, Ninth Revision, Clinical Modification [ICD-9$\mathrm{CM}$ ] or ICD-10-CM formats); (2) procedures (in ICD-9-CM, ICD-10 CM, Healthcare Common Procedure Coding System [HCPCS], or Current Procedural Terminology, fourth edition formats); (3) setting of care (e.g., inpatient or emergency department) and date[s]) of service; (4) dispensed medications from outpatient pharmacies; and (5) total reimbursed amounts for medical care and prescription 
pharmacotherapies. The data were de-identified, compliant with the Health Insurance Portability and Accountability Act of 1996 and encompassed the study period of 1 January 2003, to 31 December 2014.

\section{Patient Population}

The study population included patients with resected stage IIB, IIC, or IIIA melanoma (AJCC, seventh edition) with pathologically confirmed diagnoses between 1 January 2010, and 31 December 2013, and with evidence of resection within 4 months following diagnosis. The date of resection was designated as the index date.

Patients were excluded if they had any of the following: (1) < 12 months of enrollment in Medicare part A or part B before and after the index date; (2) an age of $<18$ years at the index date; (3) evidence of resection in the preindex period; (4) ocular/uveal melanoma or any other nonmelanoma malignancies (except nonmelanoma skin cancers and in situ cancers) in the preindex period; or (5) a record of enrollment in a health maintenance organization after the index date. The detailed sampleselection steps are outlined in Fig. 1.

The preindex period was from 2003 to the day before the index date. Patients were followed from the index date until death, disenrollment from the health plan, or end of the study period, whichever was the earliest. All available information from the SEER registry and patients' claims were compiled for the preindex period through the end of follow-up.

Ethical approval was not required for this study as data were de-identified and did not contain any identifiable patient information.

\section{Outcomes}

Patient demographics and clinical characteristics were based on information collected during the preindex baseline period, which comprised age, sex, geographic location, race, insurance type, tumor thickness, lymph node involvement, Charlson Comorbidity Index (CCI) [16], and selected comorbidities.
Outcomes of interest consisted of locoregional and distant recurrence and healthcare costs following recurrence. Since recurrence was not captured in the SEER-Medicare database, a treatment-based algorithm was used to assess recurrence, which was identified by the presence of metastases or secondary cancer (including neoplasms of skin), end-of-life care (admission to hospice care, regardless of relationship to melanoma), evidence of death (as recorded in the SEER registry or Medicare data for any cause), or cancer treatment (chemotherapy, radiotherapy, or surgery) in the post-index period following a 3-month treatment-free interval after the primary treatment [17]. The healthcare costs following recurrence were not melanoma specific, but rather were inclusive of all-cause costs designed to capture the overall real-world economic burden to the healthcare system. The total healthcare cost following recurrence incorporated the cost of hospitalization, emergency room visits, office visits, skilled nursing facilities, home health aides, hospice, and prescription medications. In all instances, reimbursed amounts (i.e., insurance reimbursement) plus patient liability (e.g., co-pay, co-insurance) served as a proxy for costs. Costs were assessed in two ways: annualized mean healthcare costs were assessed in the year following recurrence, or cumulative costs perpatient per-month (PPPM) were calculated before and after recurrence. Healthcare costs were adjusted and inflated to 2018 US dollars (USD) using the consumer price index medical component [18].

Sensitivity analyses, which excluded end-oflife care and evidence of death from the original definition of recurrence, were conducted to assess 1- and 2-year recurrence rates. For these analyses, recurrence was identified by the presence of metastases, secondary cancer, or cancer treatment in the post-index period following a 3-month treatment-free interval after the primary treatment. Patients with stage IIB and stage IIC disease were grouped together to describe baseline characteristics and cost analyses. 


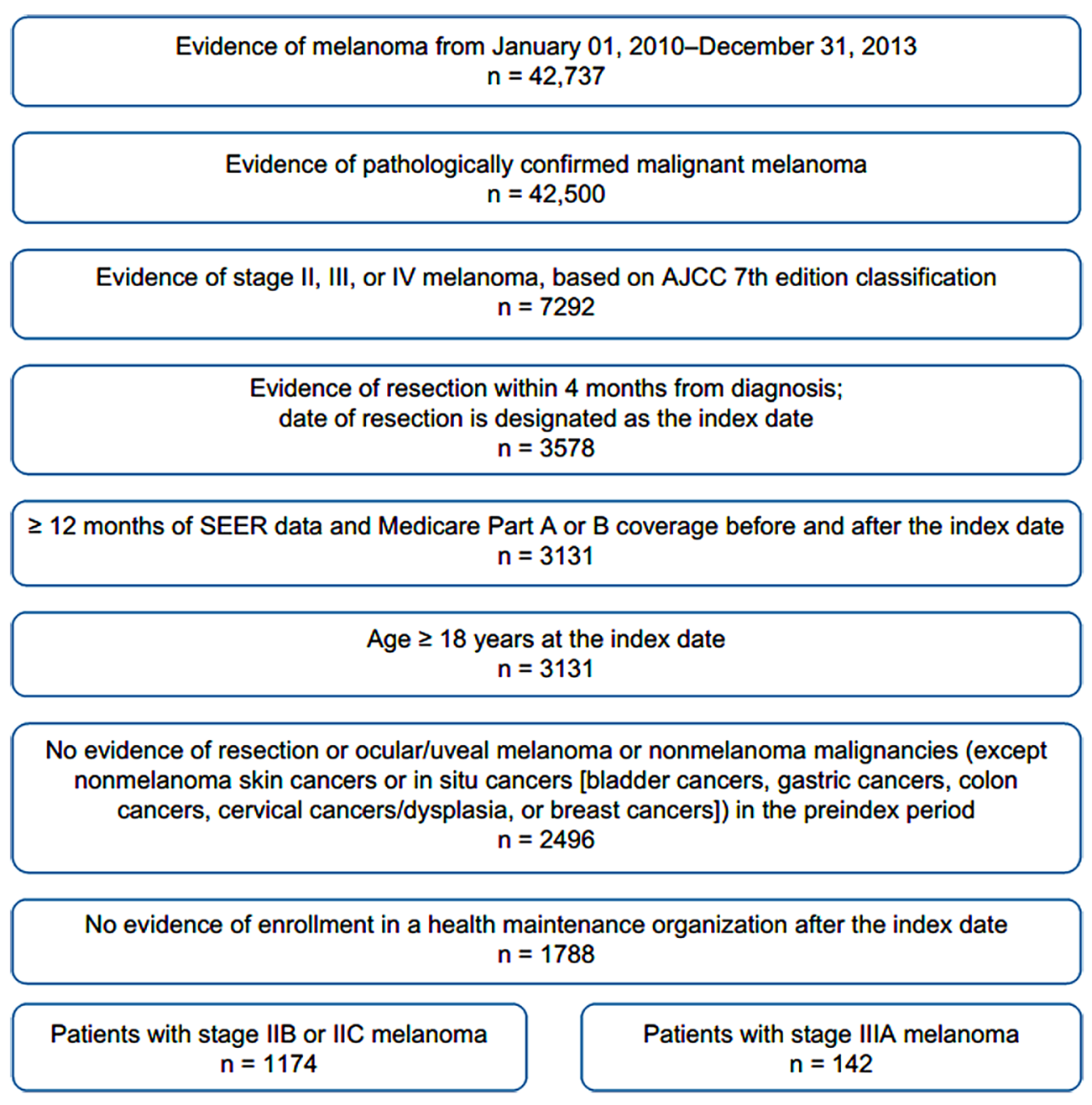

Fig. 1 Flow chart for sample selection. AJCC American Joint Committee on Cancer, SEER (US) Surveillance, Epidemiology, and End Results (program)

\section{Statistical Analyses}

Descriptive statistics were used to analyze baseline demographics and clinical characteristics. The proportion of patients who experienced recurrence during follow-up was assessed. Kaplan-Meier analyses were used to estimate RFS and OS. Costs were annualized and adjusted to reflect 2018 USD and reported as mean costs PPPM. Cumulative costs were calculated from 12 months before the first recurrence to death, patient disenrollment, or end of follow-up, whichever was earliest. For those who had a second recurrence, costs were calculated only until the second recurrence to reflect the true costs of the first recurrence only. Total healthcare costs included costs by types of medical encounter and prescription medications. Multivariate logistic regression models were conducted to estimate risk factors for recurrence. Covariates used in the models were age, sex, race, marital status, location, histologic subtype, tumor stage, ulceration, node stage, CCI, receipt of adjuvant therapy, and days to resection. All analyses were conducted using SAS version 9.4 software (SAS Institute Inc., Cary, NC, USA) and were assessed in the aggregate population as well as in subgroups. 


\section{RESULTS}

\section{Patient Characteristics}

Of the 1316 patients who met the eligibility criteria, 774 (59\%) had stage IIB, 400 (30\%) had stage IIC, and 142 (11\%) had stage IIIA melanoma. Among all patients, only 21 (2\%) received adjuvant therapy within 4 months of resection.

The mean age (standard deviation [SD]) was 79.1 (9.3) and 71.9 (11.0) years in patients with stage IIB or IIC and stage IIIA melanoma, respectively (Table 1 ). Overall, the majority of patients were male $(63 \%)$, white $(98 \%)$, and from a large metropolitan area (51\%). Comorbidities were common across patients with stage IIB or IIC and IIIA disease, including diabetes with chronic complications (16\%), congestive heart failure (9\%), and chronic pulmonary disease $(9 \%)$.

\section{Recurrence and OS}

The median durations of follow-up in patients with stage IIB, stage IIC, and stage IIIA melanoma were 28.3, 25.5, and 29.4 months, respectively. In the 1-year period following resection, $13 \%$ of patients with stage IIB, $22 \%$ with stage IIC, and $28 \%$ with stage IIIA melanoma experienced recurrence; the 2-year rates were 29,44 , and 46\%, respectively (Table 2). Median RFS (range) was 16 (4-54) months in patients with stage IIB, 15 (4-56) months in patients with stage IIC, and 10.5 (4-35) months in patients with stage IIIA melanoma. Median (range) OS was longest for patients with stage IIIA [23.5 (9-66) months], followed by stage IIB [23 (6-62) months], and stage IIC [20 (6-69) months] melanoma. Following recurrence, $49 \%$ of patients with stage IIB or IIC and $86 \%$ of those with stage IIIA melanoma had surgery for recurrence.

In the sensitivity analyses excluding end-oflife care and death as indicators of recurrence, 1 -year recurrence rates were $10 \%$ in patients with stage IIB, $17 \%$ in patients with stage IIC, and $28 \%$ in patients with stage IIIA melanoma;
2 -year recurrence rates were 24,38 , and $45 \%$, respectively.

\section{Risk Factors}

Among patients with resected stage IIB or IIC melanoma, those aged > 75 years had 85\% higher odds of having a recurrence than those aged 65-75 years [odds ratio (OR) 1.853; $95 \%$ CI $1.416,2.425]$, those with the presence of ulceration had $77 \%$ higher odds of a recurrence than those without ulceration (OR 1.771; 95\% CI 1.293, 2.425), and those with a higher incidence of comorbidities had $24 \%$ higher odds of a recurrence (OR 1.244; 95\% CI 1.129, 1.372); however, patients with T3 staging had $48 \%$ lower odds of a recurrence than those with T4 staging (OR 0.522; 95\% CI 0.393, 0.695) (Fig. 2). In patients with resected stage IIB or IIC melanoma, a multicollinearity test between tumor size ( $\mathrm{T}$ staging) and ulceration did not detect a correlation between these two factors (Pearson correlation coefficient 0.245, $P<$ 0.0001 ; data not shown); none of the patients with stage IIIA melanoma had ulceration. Among patients with resected stage IIIA melanoma, females had greater than twofold higher odds of a recurrence than males (OR 2.173; 95\% CI 1.014, 4.656); however, compared with those with nodular melanoma, those with superficial spreading and unspecified subtypes had $82 \%$ (OR $0.178 ; 95 \%$ CI $0.053,0.601)$ and 79\% (OR 0.208; 95\% CI 0.065, 0.664) lower odds of a recurrence $(P \leq 0.05)$, respectively.

\section{Costs}

In the year following recurrence until the second recurrence or the end of the follow-up period, the mean total healthcare cost (SD) was $\$ 31,870(\$ 49,147)$ in patients with stage IIB or IIC melanoma and $\$ 29,224(\$ 48,837)$ in those with stage IIIA melanoma. These costs were mainly driven by medication costs $(\$ 44,806$ in stage IIB or IIC and $\$ 84,268$ in stage IIIA melanoma) (Table 3).

Cumulative costs began to increase prior to recurrence, but they were substantially higher following recurrence for patients with stage IIB 
Table 1 Baseline demographics and clinical characteristics of patient population according to stage of melanoma

\begin{tabular}{|c|c|c|}
\hline $\begin{array}{l}\text { Baseline demographics } \\
\text { and clinical characteristics }\end{array}$ & $\begin{array}{l}\text { Stage IIB or IIC melanoma } \\
(n=1174 \text { patients })\end{array}$ & $\begin{array}{l}\text { Stage IIIA melanoma } \\
(n=142 \text { patients })\end{array}$ \\
\hline Age in years, mean $(S D)$ & $79.1(9.3)$ & $71.9(11.0)$ \\
\hline \multicolumn{3}{|l|}{ Sex, $n(\%)$} \\
\hline Male & $748(64)$ & $79(56)$ \\
\hline Female & $426(36)$ & $63(44)$ \\
\hline \multicolumn{3}{|l|}{ Race, $n(\%)$} \\
\hline White & $1152(98)$ & $142(100)$ \\
\hline Other & $22(2)$ & 0 \\
\hline \multicolumn{3}{|l|}{ Geographic location, $n(\%)$} \\
\hline West & $493(42)$ & $60(42)$ \\
\hline Northeast & $267(23)$ & $30(21)$ \\
\hline Midwest & $234(20)$ & $31(22)$ \\
\hline South & $169(14)$ & $21(15)$ \\
\hline Missing & $11(1)$ & 0 \\
\hline \multicolumn{3}{|l|}{ Metropolitan status, $n(\%)$} \\
\hline Large metropolitan & $594(51)$ & $76(54)$ \\
\hline Metropolitan/urban & $425(36)$ & $54(38)$ \\
\hline Less urban/rural & $155(13)$ & $12(8)$ \\
\hline \multicolumn{3}{|l|}{ Tumor size ${ }^{\mathrm{a}}, n(\%)$} \\
\hline $\mathrm{T} 1$ & 0 & $<11(<8)$ \\
\hline $\mathrm{T} 2$ & 0 & $<11(<8)$ \\
\hline $\mathrm{T} 3$ & $461(39)$ & $98(69)$ \\
\hline $\mathrm{T} 4$ & $713(61)$ & $>33(>23)$ \\
\hline \multicolumn{3}{|l|}{ Ulceration, $n(\%)$} \\
\hline Yes & $860(73)$ & NA \\
\hline No & $312(27)$ & NA \\
\hline \multicolumn{3}{|l|}{$\mathrm{N}$ staging ${ }^{\mathrm{a}}, n(\%)$} \\
\hline No & $1174(100)$ & 0 \\
\hline N1 & 0 & $123(87)$ \\
\hline $\mathrm{N} 2$ & 0 & $19(13)$ \\
\hline \multicolumn{3}{|l|}{ Histologic subtype, $n$ (\%) } \\
\hline Nodular & $399(34)$ & $25(18)$ \\
\hline Superficial spreading & $140(12)$ & $43(30)$ \\
\hline
\end{tabular}


Table 1 continued

\begin{tabular}{lll}
\hline $\begin{array}{l}\text { Baseline demographics } \\
\text { and clinical characteristics }\end{array}$ & $\begin{array}{l}\text { Stage IIB or IIC melanoma } \\
(\boldsymbol{n}=\mathbf{1 1 7 4} \text { patients })\end{array}$ & $\begin{array}{l}\text { Stage IIIA melanoma } \\
(\boldsymbol{n}=\mathbf{1 4 2} \text { patients })\end{array}$ \\
\hline Lentigo maligna & $34(3)$ & 0 \\
Acral lentiginous & $28(2)$ & $<11(<8)$ \\
Amelanotic & $12(1)$ & $<11(<8)$ \\
Other uncommon & $150(13)$ & $<11(<8)$ \\
Unspecified & $411(35)$ & $64(45)$ \\
CCI, mean (SD) & $0.7(1.3)$ & $0.5(1.2)$ \\
Comorbidities, $n$ (\%) & $198(17)$ & $19(13)$ \\
Diabetes (with chronic complications) & $109(9)$ & $<11(<8)$ \\
Congestive heart failure & $104(9)$ & $<11(<8)$ \\
Chronic pulmonary disease & $83(7)$ & $<11(<8)$ \\
Renal disease & $49(4)$ & $<11(<8)$ \\
Myocardial infarction & $44(4)$ & $<11(<8)$ \\
Cerebrovascular disease & $35(3)$ & $<11(<8)$ \\
Peripheral vascular disease & $21(2)$ & $<11(<8)$ \\
Rheumatic disease & $15(1)$ & $<11(<8)$ \\
Autoimmune disease & $S D(2 n)$ & \\
\hline
\end{tabular}

$C C I$ Charlson Comorbidity Index, $N A$ not applicable, $S D$ standard deviation

${ }^{a}$ American Joint Committee on Cancer, seventh edition [7]

or IIC and IIIA melanoma. In patients with stage IIB or IIC melanoma, the total mean cumulative cost was \$2254 PPPM at 12 months prior to recurrence; costs in the post-recurrence period were $\$ 41,093$ at 6 months, $\$ 74,225$ at 12 months, and $\$ 310,953$ at 48 months (Fig. 3). The trend was similar in patients with stage IIIA melanoma, among whom the total mean cumulative costs were $\$ 1976$ PPPM at 12 months prior to recurrence and $\$ 30,895$, $\$ 52,971$, and $\$ 166,060$ at 6,12 , and 48 months, respectively, in the post-recurrence period. In both groups, cancer therapy was the major driver of cost, followed by outpatient and inpatient costs.

\section{DISCUSSION}

To our knowledge, this is the first populationbased, real-world study assessing recurrence rates, risk factors for recurrence, and costs of recurrence in patients with resected stage IIB or IIC and IIIA melanoma. Using a treatmentbased algorithm, we have shown that more than one-third of patients with resected stage IIB or IIC melanoma and nearly one-half of those with resected stage IIIA melanoma experienced recurrence within 2 years. These results were consistent with those from an earlier German registry study that recorded recurrence in $13.8 \%$ of patients with stage II and $28.2 \%$ of those with stage III melanoma at 1 year; $31.7 \%$ and $48.0 \%$ of patients, respectively, experienced 
Table 2 Clinical outcomes of patient population according to stage of melanoma

\begin{tabular}{|c|c|c|c|}
\hline Outcomes & $\begin{array}{l}\text { Stage IIB melanoma } \\
(n=774 \text { patients })\end{array}$ & $\begin{array}{l}\text { Stage IIC melanoma } \\
(n=400 \text { patients })\end{array}$ & $\begin{array}{l}\text { Stage IIIA melanoma } \\
(n=142 \text { patients })\end{array}$ \\
\hline 1 -year recurrence, $n(\%)$ & $97(13)$ & $87(22)$ & $40(28)$ \\
\hline 2-year recurrence, $n(\%)$ & $227(29)$ & $174(44)$ & $66(46)$ \\
\hline \multicolumn{4}{|c|}{ Recurrence-free survival, months } \\
\hline Mean (SD) & $19.2(0.5)$ & $16.9(0.7)$ & $13.5(0.8)$ \\
\hline Median (range) & $16(4-54)$ & $15(4-56)$ & $10.5(4-35)$ \\
\hline \multicolumn{4}{|c|}{ Distant metastasis-free survival, months } \\
\hline Mean (SD) & $18.9(0.9)$ & $17.0(0.8)$ & $14.6(1.0)$ \\
\hline Median (range) & $16(4-52)$ & $17(4-45)$ & $12(4-37)$ \\
\hline \multicolumn{4}{|l|}{ Overall survival, months } \\
\hline Mean (SD) & $25.7(0.8)$ & $23.1(0.9)$ & $27.6(2.3)$ \\
\hline Median (range) & $23(6-62)$ & $20(6-69)$ & $23.5(9-66)$ \\
\hline
\end{tabular}

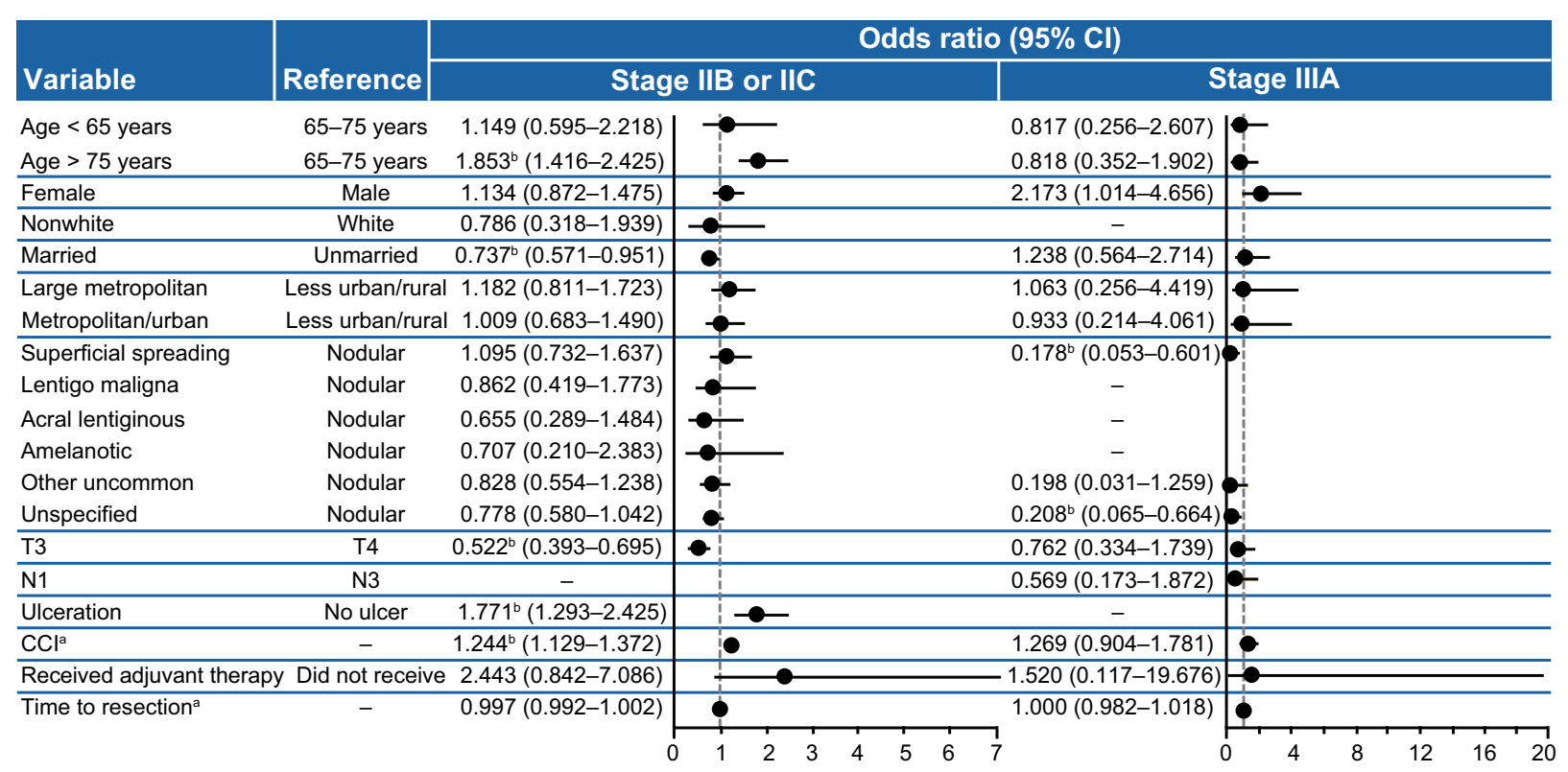

Fig. 2 Resected stage IIB or IIC and stage IIIA melanoma. Covariates were included in the model for assessment of risk factors for recurrence. ${ }^{\mathrm{a} C}$ Continuous variable; ${ }^{\mathrm{b}} P \leq 0.05$. CCI Charlson Comorbidity Index, $C I$ confidence interval

recurrence at 3 years [10]. Another study reported recurrence in $48 \%$ of patients with resected stage IIIA melanoma at 5 years; however, this earlier study used older data (1992-2004) from a single site [19]. In contrast, one study reported a recurrence rate of $19 \%$ at a mean follow-up of 23.1 months [20], which was lower than that reported in our study. This may have been due to the inclusion of a younger patient population (mean age 53.8 years) with stage I and IIA 
Table 3 Annual healthcare costs in the year following recurrence (2018 USD)

\begin{tabular}{|c|c|c|}
\hline Annualized costs (2018 USD) & Stage IIB or IIC melanoma & Stage IIIA melanoma \\
\hline Hospital visit & $n=130$ & $n=20$ \\
\hline Mean (SD) & $21,313(37,101)$ & $13,654(16,467)$ \\
\hline Median (range) & $10,068(25-305,328)$ & $8440(15-64,263)$ \\
\hline Emergency room visit & $n=158$ & $n=27$ \\
\hline Mean (SD) & $936(1130)$ & $1007(1101)$ \\
\hline Median (range) & $550(38-10,758)$ & $640(26-5406)$ \\
\hline Office visit & $n=242$ & $n=60$ \\
\hline Mean (SD) & $15,968(31,908)$ & $18,890(42,085)$ \\
\hline Median (range) & $3772(18-183,846)$ & $4743(21-232,780)$ \\
\hline Radiation therapy & $n=109$ & $n=20$ \\
\hline Mean (SD) & $23,107(38,302)$ & $23,260(32,532)$ \\
\hline Median (range) & $10,805(307-250,321)$ & $13,035(480-128,447)$ \\
\hline Surgery & $n=265$ & $n=64$ \\
\hline Mean (SD) & $10,036(29,268)$ & $8124(18,163)$ \\
\hline Median (range) & $2559(7-397,587)$ & $3017(8-139,177)$ \\
\hline Cancer medication & $n=174$ & $n=37$ \\
\hline Mean (SD) & $108,532(166,065)$ & $153,913(261,511)$ \\
\hline Median (range) & $38,285(1-956,147)$ & $43,993(2-1,320,640)$ \\
\hline Noncancer medication & $n=500$ & $n=74$ \\
\hline Mean (SD) & $14,262(45,727)$ & $20,099(44,752)$ \\
\hline Median (range) & $2385(5-515,364)$ & $4567(77-272,153)$ \\
\hline Skilled nursing facility & $n=76$ & $n<11$ \\
\hline Mean (SD) & $12,304(22,557)$ & $25,297(33,439)$ \\
\hline Median (range) & $5129(89-140,873)$ & $11,877(71-85,929)$ \\
\hline Home healthcare & $n=62$ & $n<11$ \\
\hline Mean (SD) & $3231(5578)$ & $3986(5477)$ \\
\hline Median (range) & $1343(64-28,724)$ & $1594(641-16,119)$ \\
\hline Hospice & $n=125$ & $n=18$ \\
\hline Mean (SD) & $8593(15,612)$ & $10,038(19,355)$ \\
\hline Median (range) & $2947(218-95,694)$ & $4194(294-83,884)$ \\
\hline Total healthcare $^{\mathrm{a}}$ & $n=534$ & $n=74$ \\
\hline Mean (SD) & $31,870(49,147)$ & $29,224(48,837)$ \\
\hline
\end{tabular}


Table 3 continued

\begin{tabular}{lcc}
\hline Annualized costs (2018 USD) & Stage IIB or IIC melanoma & Stage IIIA melanoma \\
\hline Median (range) & $14,028(83-333,964)$ & $10,779(21-258,532)$ \\
\hline $\begin{array}{l}\text { a Defined as the sum of costs for hospital visit(s), office visit(s), medication(s) (Medicare part D only), skilled nursing } \\
\text { facility, home healthcare, and hospice }\end{array}$
\end{tabular}

melanoma who have a lower risk of recurrence [20]. The current study highlights the risk of recurrence in patients with resected early-stage melanoma. Future clinical trials are needed to understand whether these patients will benefit from adjuvant therapies, such as immune checkpoint inhibitors [3,5] or BRAF plus MEK inhibitors [4].

In this study, we identified patient subgroups with a high risk of recurrence (older patients, those with ulceration, those with more advanced disease, and those with nodular melanoma), which may help clinicians distinguish patients who are likely to benefit from newer adjuvant therapies. A recent single-center cohort study also showed that recurrences and metastases were influenced by patient age [21]. Although tumor size and ulceration are associated with a high risk of recurrence, a correlation between these two factors was not found in the current study.

Furthermore, the high recurrence rates in patients with resected stage IIB or IIC and IIIA melanoma resulted in substantial costs in the long term. Mean healthcare costs in the year following recurrence were similar in patients with resected stage IIB or IIC $(\$ 31,870)$ and IIIA $(\$ 29,224)$ melanoma. In contrast, a previous study reported annual estimated costs of $\$ 10,000$ for local/regional recurrence and $\$ 180,000$ for distant metastasis in patients with resected melanoma [20]. While our study population differed from that of this earlier study, annual recurrence costs in our study fell within the range of local/regional recurrence and distant metastasis costs identified earlier. Several other studies have reported direct medical costs following melanoma diagnosis; however, these studies used different patient populations and differed in methodology $[22,23]$.
The mean healthcare cost in our study was slightly higher in patients with stage IIB or IIC melanoma than in those with stage IIIA melanoma, which may be attributed to the relatively older patient population (79.1 vs. 71.9 years) with a higher comorbidity burden (0.67 vs. 0.50 ) in the former group. The mean cumulative cost PPPM until recurrence was $\$ 6730$ for stage IIB or IIC melanoma versus $\$ 4406$ for stage IIIA melanoma. Another possible reason for the higher costs in patients with stage IIB or IIC melanoma may have been the lower proportion of patients who had surgery for recurrence compared with those with stage IIIA melanoma (49 vs. $86 \%$ ), and surgery is known to lower follow-up costs and prolong survival [24-26]. These factors may also have contributed to the slightly longer OS in patients with stage IIIA melanoma than in those with stage IIB and IIC melanoma (23.5 vs. 23 and 20 months, respectively).

Our study has several potential limitations. First, a treatment-based algorithm and proxies such as "all-cause" death and end-of-life care were used to identify probable recurrences during follow-up. Some patients did not receive treatment upon recurrence, which may have led to an underestimation of the rate of recurrence and associated costs. On the other hand, proxies such as death or end-of-life care may have led to an overestimation of the rate of recurrence. Second, this study was based on analyses of registries and claims data, which may have been subject to errors of omission and/or commission. Third, while cost estimates represented the total amount paid to providers for services and prescription medications, they did not include indirect costs. Finally, while nationally representative, the study was a convenience sample that included only patients within the SEER registry with Medicare coverage. While the SEER 
a

Stage IIB or IIC

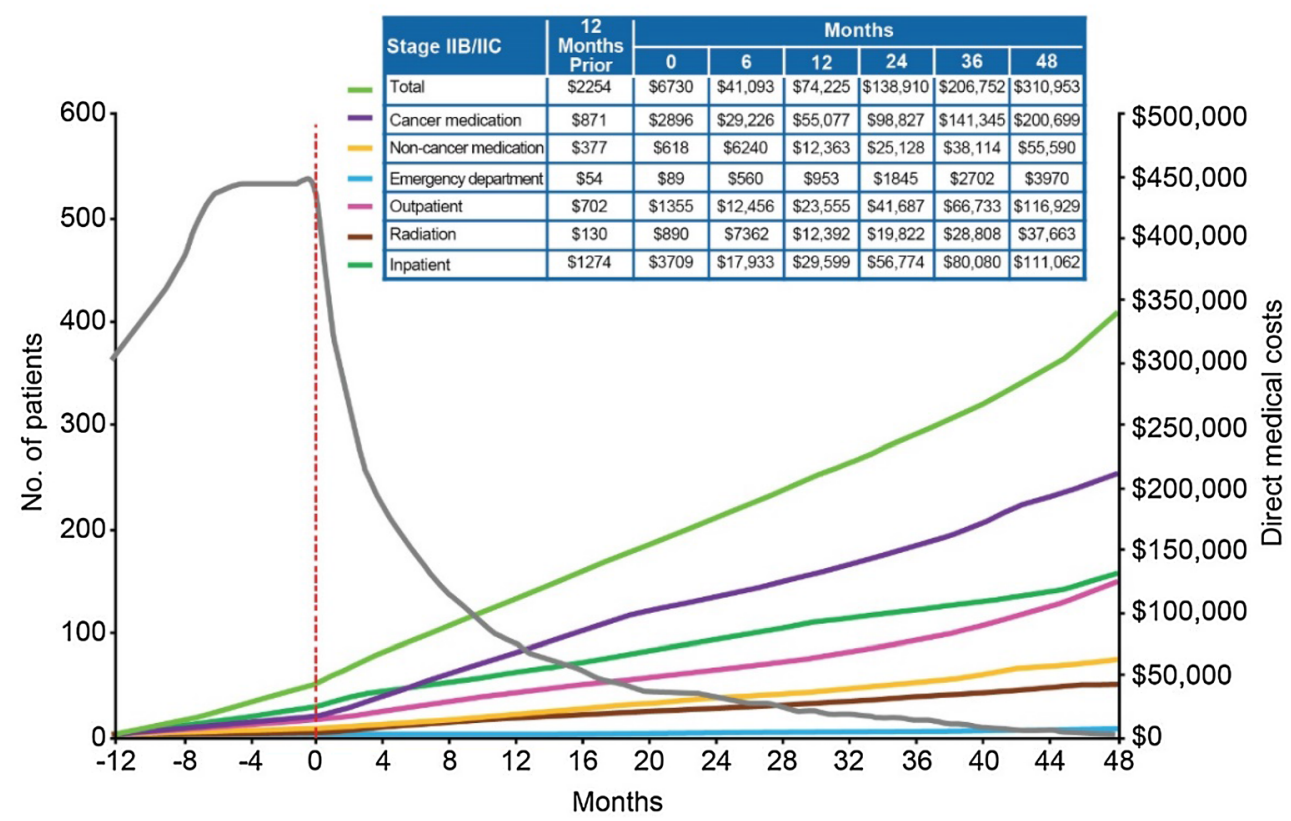

b

Stage IIIA

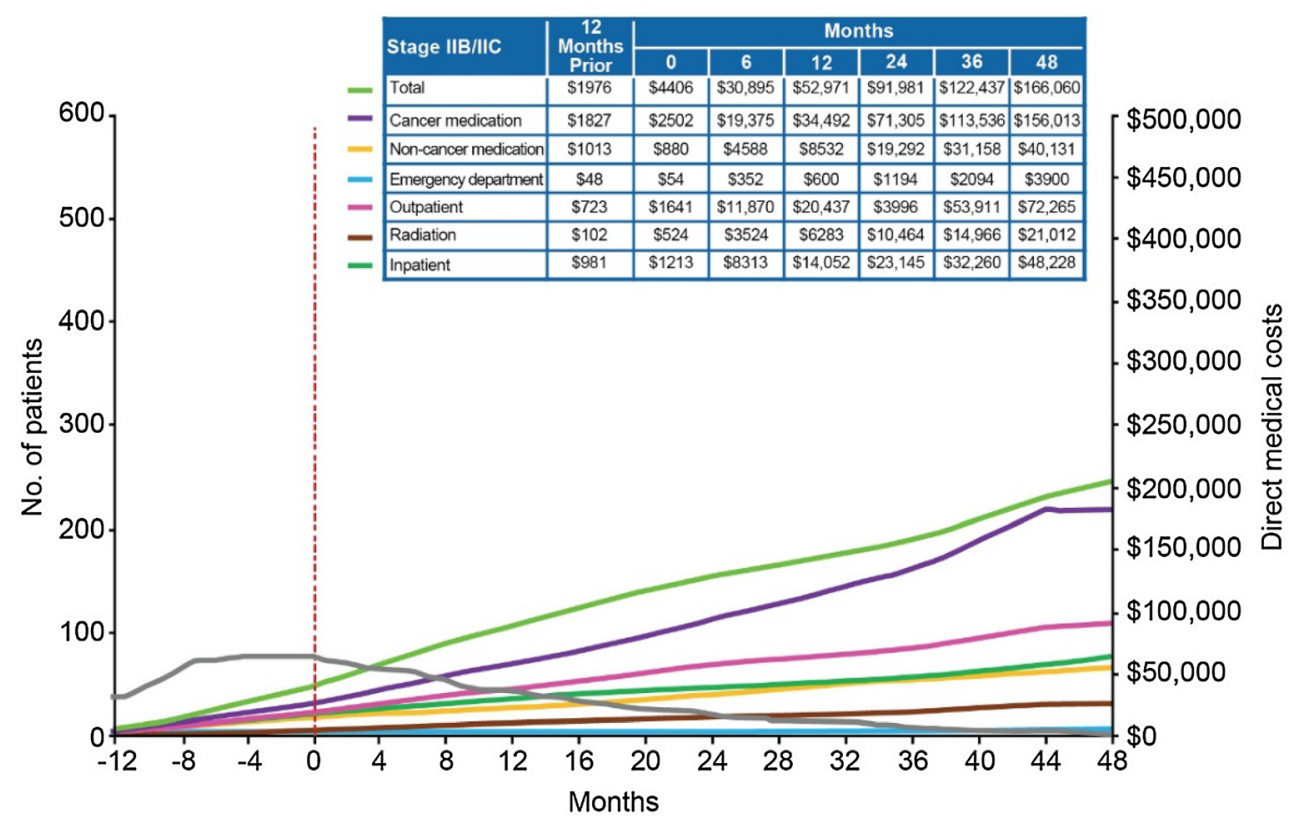

Fig. 3 Resected stage IIB or IIC (a) and stage IIIA (b) melanoma. Cumulative costs per patient per month after recurrence. The vertical red dotted line represents first recurrence; the gray line represents the sample size

data cover about $14 \%$ of all cancer cases, certain groups are underrepresented, such as African Americans and other races, and hence the study may not be generalizable to all patients with melanoma in the USA.

\section{CONCLUSIONS}

There is a scarcity of evidence on recurrence rates in early-stage melanoma. This real-world study showed that approximately one-third of 
patients with stage IIB or IIC melanoma and nearly one-half of those with stage IIIA melanoma experienced a recurrence within 2 years following resection, which was associated with high healthcare costs. Further research is required to understand how the benefits of recently approved adjuvant therapies can reduce recurrence and associated costs in this patient population.

\section{ACKNOWLEDGEMENTS}

This study used the linked SEER-Medicare database. The interpretation and reporting of these data are the sole responsibility of the authors. The authors acknowledge the efforts of the National Cancer Institute; the Office of Research, Development and Information, CMS; Information Management Services (IMS), Inc.; and the Surveillance, Epidemiology, and End Results (SEER) Program tumor registries in the creation of the SEER-Medicare database.

Funding. Sponsorship for this study and the journal's Rapid Service Fee were funded by Bristol Myers Squibb, Princeton, NJ, USA.

Editorial Assistance. Editorial assistance was provided by Kakoli Parai, PhD, and Andrea Lockett of Ashfield Healthcare Communications, funded by Bristol Myers Squibb.

Authorship. All named authors meet the International Committee of Medical Journal Editors (ICMJE) criteria for authorship for this article, take responsibility for the integrity of the work as a whole, and have given their approval for this version to be published.

Authorship Contributions. Sekwon Jang, Tayla Poretta, Tarun Bhagnani, Matthew Burke, and Sumati Rao were involved in the conception of this study and the study design. Tarun Bhagnani and Qing Harshaw participated in acquisition and collection of data and conducted the statistical analyses. Sekwon Jang, Tayla Poretta, Tarun Bhagnani, Matthew Burke, and Sumati Rao reviewed the data and interpreted the findings of this study.

Prior Presentation. Data from this study were presented in part at the 16th International Congress of the Society for Melanoma Research (SMR), held November 20-23, 2019, in Salt Lake City, UT, USA.

Disclosures. Sekwon Jang reports personal fees from Bristol Myers Squibb during the conduct of the study. Tarun Bhagnani and Qing Harshaw are employees of EPI-Q Inc., which received payment from Bristol Myers Squibb for conducting the study. Tayla Poretta is an employee of Bristol Myers Squibb. Matthew Burke and Sumati Rao are employees of and stockholders in Bristol Myers Squibb.

Compliance with Ethics Guidelines. Ethical approval was not required for this study as data were de-identified and did not contain any identifiable patient information.

Data Availability. The Bristol Myers Squibb policy on data sharing may be found at https:// www.bms.com/researchers-and-partners/independ ent-research/data-sharing-request-process.html.

Open Access. This article is licensed under a Creative Commons Attribution-NonCommercial 4.0 International License, which permits any non-commercial use, sharing, adaptation, distribution and reproduction in any medium or format, as long as you give appropriate credit to the original author(s) and the source, provide a link to the Creative Commons licence, and indicate if changes were made. The images or other third party material in this article are included in the article's Creative Commons licence, unless indicated otherwise in a credit line to the material. If material is not included in the article's Creative Commons licence and your intended use is not permitted by statutory regulation or exceeds the permitted use, you will need to obtain permission directly from the copyright holder. To view a copy of this licence, visit http://creativecommons.org/licenses/bync/4.0/. 


\section{REFERENCES}

1. US Cancer Statistics Working Group. US Cancer Statistics Data Visualizations Tool, based on November 2018 submission data (1999-2016): US Department of Health and Human Services, Centers for Disease Control and Prevention and National Cancer Institute. 2019. https://gis.cdc.gov/Cancer/ USCS/DataViz.html. Accessed November 2019.

2. National Comprehensive Cancer Network ${ }^{\circledR}$. NCCN Clinical Practice Guidelines in Oncology (NCCN Guidelines ${ }^{\circledR}$ ): Cutaneous Melanoma Version 1 . $2020 . \quad$ https://www.nccn.org/professionals/ physician_gls/pdf/cutaneous_melanoma.pdf. Accessed Jan 2020.

3. Weber J, Mandala M, Del Vecchio M, et al. Adjuvant nivolumab versus ipilimumab in resected stage III or IV melanoma. N Engl J Med. 2017;377(19): 1824-35.

4. Long GV, Hauschild A, Santinami M, et al. Adjuvant dabrafenib plus trametinib in stage III BRAFmutated melanoma. N Engl J Med. 2017;377(19): 1813-23.

5. Eggermont AMM, Blank CU, Mandala M, et al. Adjuvant pembrolizumab versus placebo in resected stage III melanoma. $\mathrm{N}$ Engl J Med. 2018;378(19):1789-801.

6. Balch CM, Gershenwald JE, Soong SJ, et al. Final version of 2009 AJCC melanoma staging and classification. J Clin Oncol. 2009;27(36):6199-206.

7. Gershenwald JE, Scolyer RA, Hess KR, et al. Melanoma staging: evidence-based changes in the American Joint Committee on Cancer Eighth Edition Cancer Staging Manual. CA Cancer J Clin. 2017;67:472-92.

8. Bajaj S, Donnelly D, Call $\mathrm{M}$, et al. Melanoma prognosis accuracy of the American Joint Committee on Cancer Staging Manual Eighth Edition. J Natl Cancer Inst. 2020. https://doi.org/10.1093/ jnci/djaa008.

9. Bleicher J, Swords DS, Bowles TL, Hyngstrom JR. Patterns of recurrence in stage II cutaneous melanoma. Ann Surg Oncol. 2019;26:S160 (Abstract P273).

10. Leiter U, Buettner PG, Eigentler TK, et al. Hazard rates for recurrent and secondary cutaneous melanoma: an analysis of 33,384 patients in the German Central Malignant Melanoma Registry. J Am Acad Dermatol. 2012;66:37-45.

11. Melanoma Research Alliance. Interferon (Intron ${ }^{\circledR} \mathrm{A}$ or Sylatron ${ }^{\mathrm{TM}}$ ). https://www.curemelanoma.org/ patient-eng/melanoma-treatment/adjuvant-therapy/in terferon-intron-a-or-sylatron/. 2016. Accessed December 2019.

12. Kirkwood JM, Strawderman MH, Ernstoff MS, et al. Interferon alfa-2b adjuvant therapy of high-risk resected cutaneous melanoma: the Eastern Cooperative Oncology Group Trial EST 1684. J Clin Oncol. 1996;14(1):7-17.

13. Kirkwood JM, Ibrahim JG, Sondak VK, et al. Highand low-dose interferon alfa-2b in high-risk melanoma: first analysis of intergroup trial E1690/ S9111/C9190. J Clin Oncol. 2000;18(12):2444-58.

14. Agarwala SS, Lee SJ, Flaherty LE, et al. Randomized phase III trial of high-dose interferon alfa-2b (HDI) for 4 weeks induction only in patients with intermediate- and high-risk melanoma (Intergroup trial E 1697). J Clin Oncol. 2011;29(15 Suppl):8505.

15. National Cancer Institute, Division of Cancer Control and Population Sciences. Surveillance, Epidemiology, and End Results (SEER). 2018. https://seer.cancer.gov/about/factsheets/SEER_Ove rview.pdf. Accessed Nov 2019.

16. Quan H, Sundararajan V, Halfon P, et al. Coding algorithms for defining comorbidities in ICD-9-CM and ICD-10 administrative data. Med Care. 2005;43(11):1130-9.

17. Warren JL, Mariotto A, Melbert D, et al. Sensitivity of Medicare claims to identify cancer recurrence in elderly colorectal and breast cancer patients. Med Care. 2016;54(8):e47-54.

18. US Department of Health and Human Services. Health Resources and Services Administration (HRSA). Consumer price index for medical care. $2018 . \quad$ https://www.hrsa.gov/get-health-care/ affordable/hill-burton/cpi.html. Accessed March 2020.

19. Romano E, Scordo M, Dusza SW, et al. Site and timing of first relapse in stage III melanoma patients: implications for follow-up guidelines. J Clin Oncol. 2010;28(18):3042-7.

20. Tarhini A, Ghate SR, Ionescu-Ittu R, et al. Postsurgical treatment landscape and economic burden of locoregional and distant recurrence in patients with operable nonmetastatic melanoma. Melanoma Res. 2018;28(6):618-28.

21. Gassenmaier M, Keim U, Leiter U, et al. Age as key factor for pattern, timing and extent of distant metastasis in patients with cutaneous melanoma: a study of the German Central Malignant Melanoma Registry. J Am Acad Dermatol. 2019;80:1299-307. 
22. Seidler AM, Pennie ML, Veledar E, et al. Economic burden of melanoma in the elderly population: population-based analysis of the Surveillance, Epidemiology, and End Results (SEER)-Medicare data. Arch Dermatol. 2010;146(3):249-56.

23. Davis KL, Mitra D, Kotapati S, et al. Direct economic burden of high-risk and metastatic melanoma in the elderly: evidence from the SEER-Medicare linked database. Appl Health Econ Health Policy. 2009; 7(1):31-41.

24. Buja A, Sartor G, Scioni M, et al. Estimation of direct melanoma-related costs by disease stage and by phase of diagnosis and treatment according to clinical guidelines. Acta Derm Venereol. 2018;98(2):218-24.

25. Sosman JA, Moon J, Tuthill RJ, et al. A phase 2 trial of complete resection for stage IV melanoma: results of Southwest Oncology Group Clinical Trial S9430. Cancer. 2011;117(20):4740-6.

26. Howard JH, Thompson JF, Mozzillo N, et al. Metastasectomy for distant metastatic melanoma: analysis of data from the first Multicenter Selective Lymphadenectomy Trial (MSLT-I). Ann Surg Oncol. 2012;19(8):2547-55. 